\title{
Prediction of breakdown strength of cellulosic insulating materials using artificial neural networks
}

\author{
Sakshi Singh*, M. M. Mohsin and Aejaz Masood \\ Department of Electrical Engineering \\ Z. H. College of Engineering \& Technology \\ Aligarh Muslim University, Aligarh, UP 202002, India \\ *sakshisingh3009@gmail.com
}

Received 3 November 2017; Revised 24 January 2018; Accepted 26 January 2018; Published 26 February 2018

\begin{abstract}
In this research work, a few sets of experiments have been performed in high voltage laboratory on various cellulosic insulating materials like diamond-dotted paper, paper phenolic sheets, cotton phenolic sheets, leatheroid, and presspaper, to measure different electrical parameters like breakdown strength, relative permittivity, loss tangent, etc. Considering the dependency of breakdown strength on other physical parameters, different Artificial Neural Network (ANN) models are proposed for the prediction of breakdown strength. The ANN model results are compared with those obtained experimentally and also with the values already predicted from an empirical relation suggested by Swanson and Dall. The reported results indicated that the breakdown strength predicted from the ANN model is in good agreement with the experimental values.
\end{abstract}

Keywords: Cellulosic materials; relative permittivity; loss tangent; breakdown strength; ANN.

\section{Introduction}

A large number of solid insulating materials are used in electric power industry. Cellulose insulation has been a preferred choice in power equipment since it is available abundantly from natural resources like soft wood, cotton, silk, etc. However, cellulosic materials are hygroscopic in nature and need to be used in dry conditions. This is the main disadvantage associated with the use of cellulosic material in electrical machines. With time, advances in the field of cellulosic material have been made and material available nowadays shows acceptable thermal and mechanical strength for present day applications, which is achieved by the use of better pulps, optimized fiber length, exemplary modern binders and overall thermal upgrading of the material. ${ }^{1-3}$

When electric field is applied to any ideal insulating material, it should not exhibit any electrical conductivity. However, they all have some conductivity, which changes with environmental and field conditions. If the applied field increases to some critical magnitude, material can break down electrically and large current flow could result in failure of electrical equipment. This field is called the breakdown strength or dielectric strength of the insulating material. It depends on the insulating material properties and should be measured under specified and controlled experimental conditions. It is very important to concern about the electrical failure of insulating material operating at high voltage and proper analysis is necessary to estimate the life and performance of the material before its practical use. ${ }^{1-5}$

According to Swanson and Dall, a correlation can be obtained between parameter thickness $t$, volume resistivity
" $\rho_{v}$ ", relative permittivity $\xi_{r}$, and loss tangent $\tan \delta$ and dielectric strength. They proposed a relationship which can be useful for predicting the breakdown strength of any electrical solid insulating material. ${ }^{4}$ The suggested approach has been used in a previous work for the same cellulosic insulation samples and a relationship has been proposed to predict the breakdown strength. The result reported in that work was found to be in good agreement with the measured value. ${ }^{6}$

The present work is focused on the prediction of breakdown strength as a function of other physical parameters using a nature-inspired computational technique, that is, Artificial Neural Networks (ANNs). Breakdown strength, relative permittivity, and loss tangent are measured by performing different experiments in high voltage laboratory. The data acquired from the experiments are analyzed to propose a simulation model using ANN for the prediction of breakdown strength. Also, the results obtained from ANN model are compared with the results of previous work to show them with the experimentally measured value.

\section{Experimental Techniques}

The experiments have been performed on different cellulosic materials like diamond-dotted paper, paper phenolic sheets, cotton phenolic sheets, leatheroid, and presspaper. The materials were tested as available in the laboratory. ${ }^{7}$ Value of volume resistivity for each sample is taken from the literature or as provided by the manufacturer. ${ }^{8-10}$

Measurement of $\xi_{r}$ and $\tan \delta$ is carried out by placing materials under observation between the parallel plates of

This is an Open Access article published by World Scientific Publishing Company. It is distributed under the terms of the Creative Commons Attribution 4.0 (CC-BY) License. Further distribution of this work is permitted, provided the original work is properly cited. 


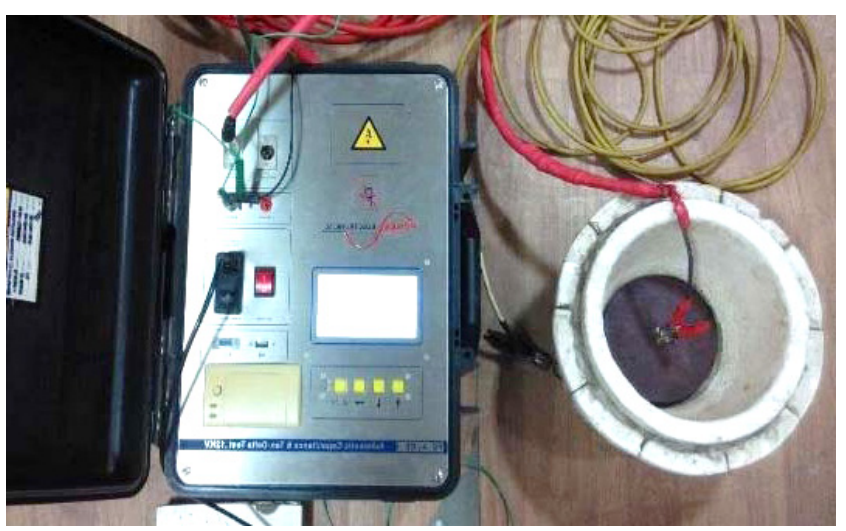

Fig. 1. Parallel plate electrode cell with ACDF-1 present in HV lab.

ASTM electrode assembly, ${ }^{11}$ with the help of an automatic capacitance and tan delta (dissipation factor) bridge. ${ }^{12,13}$ Experimental setup with connections is shown in Fig. 1. Capacitance $(C)$ obtained from ACDF bridge is used to calculate $\xi_{r}$ using the following equation:

$$
\xi_{r}=(t * C) /\left(A * \xi_{0}\right),
$$

where $A$ is the surface area of electrode and $\xi_{0}$ is the permittivity of free space $\left(8.8542 \times 10^{-12} \mathrm{~F} / \mathrm{m}\right)$.

To measure breakdown voltage, ASTM point to plane electrode assembly is used. Electrode assembly and experimental setup is shown in Fig. 2. High voltage is applied to the point to plain electrode system using a $150 \mathrm{kV}, 50 \mathrm{~Hz}$ high voltage transformer. ${ }^{14,15}$ Breakdown strength can be calculated from the observed values of breakdown voltage from the relation:

\section{Breakdown Strength $=($ Breakdown Voltage $(\mathrm{kV})) /$} (Thickness of insulation $(\mathrm{mm})$ ).

Experimental techniques and measured experimental data are given in more details in Ref. 6.

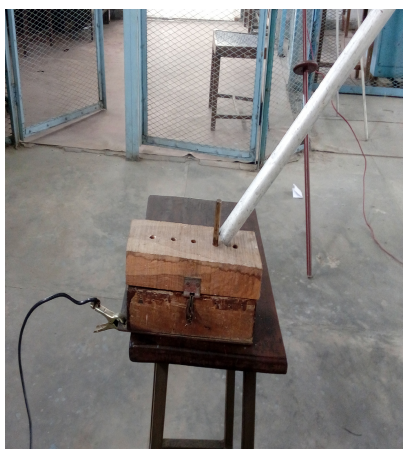

(a)

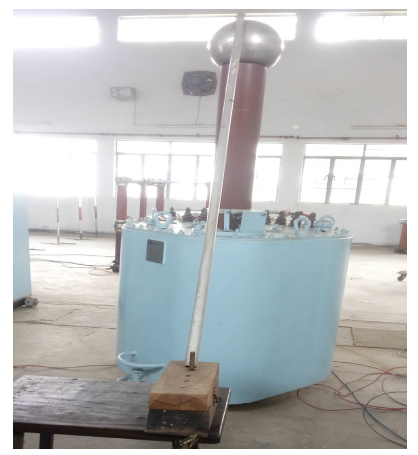

(b)
Fig. 2. Electrode assembly to measure breakdown strength present in HV lab.

\section{Prediction of Breakdown Strength Using ANN}

\subsection{Background}

ANN is a computational technique inspired by the human biological neural networks structure and function similarly as human brain does by memorizing and learning various jobs. It is used to predict system performance or output mathematically by predicting certain behavior and to recollect that behavior whenever required in future similar to human brain. ${ }^{16}$

In this work, ANN toolbox of MATLAB (R2013a) is used to prepare different ANN models. ${ }^{17}$ Thickness of insulation, relative permittivity, loss tangent, and volume resistivity are used as inputs and breakdown strength is used as target. Multilayer feedforward network is provided with input-output data set and trained using the back-propagation algorithm. Other configurations taken to prepare a model are tabulated in Table 1.

Each input or output data are normalized as $P_{i}$ according to the formula:

$$
P_{i}=x+(y-x)\left(X_{i}-X_{\min }\right) /\left(X_{\max }-X_{\min }\right),
$$

where $i=1,2, \ldots, n, P_{i}=$ normalized value; $x=$ lower limit of normalized value $=0.1 ; y=$ upper limit of normalized value $=0.9, X_{i}=$ value of data to be normalized; $X_{\min }=$ minimum value in the data set; $X_{\max }=$ maximum value in the data set.

\subsection{Results}

Training and testing data for ANN model are taken from experimental readings as well as the literature. Every model is trained and validated using 233 data pairs and then trained model is tested using 11 data pairs for cellulosic materials.

ANN model with one, two, and three hidden layers with varying number of neurons in them was trained and tested to achieve best performance. The one having best performance is selected for prediction of breakdown strength. Also, the correlation coefficient $(R)$ measures how precisely the ANN model outputs follow the desired output and it should be closer to 1 .

After training several models, it is found that the ANN architectures like 4-5-34-1 (4 neurons in input layer, two hidden layers with 5 and 34 neurons, and 1 in output layer),

Table 1. Configurations of ANN model.

\begin{tabular}{ll}
\hline ANN parameters & \multicolumn{1}{c}{ Specification } \\
\hline Transfer function & $\begin{array}{l}\text { Log-sigmoid (for hidden layers) } \\
\text { Pure-linear (for output layer) }\end{array}$ \\
Performance function & $\begin{array}{l}\text { MSE (for training) } \\
\text { MAE (for testing) }\end{array}$ \\
Training algorithm & Levenberg-Marquardt algorithm \\
No. of epochs & 1000 \\
\hline
\end{tabular}




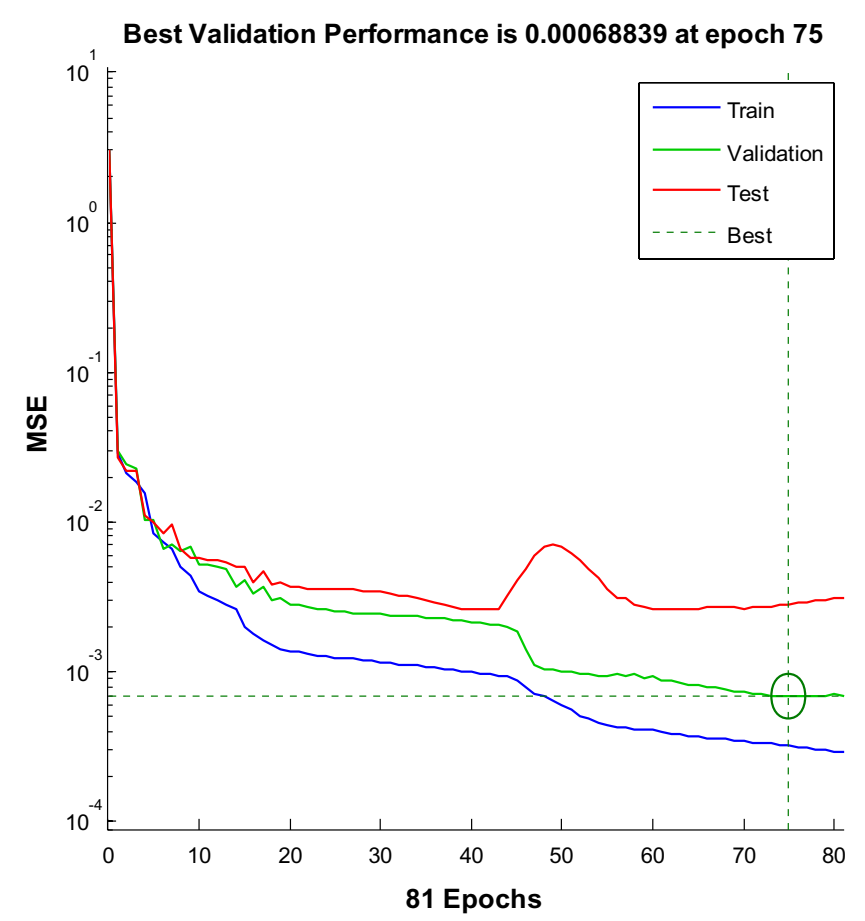

Fig. 3. Performance plot for ANN architecture 4-7-5-13-1.

4-5-34-1 (4 neurons in input layer, two hidden layers with 5 and 34 neurons, 1 neuron in output layer), 4-7-14-1 (4 neurons in input layer, two hidden layers with 7 and 14 neurons, 1 output layer neuron), and 4-7-5-13-1 (4 neurons in input layer, three hidden layers with 7,5 , and 13 neurons, 1 neuron in output layer), show good performance. Best one among them is 4-7-5-13-1 with the lowest mean absolute error (MAE) that is 1.29 and is further used for the prediction of breakdown strength of materials under study in this work.

The performance plot of 4-7-5-13-1 ANN architecture is shown in Fig. 3. It is observed that the best validation performance in terms of mean square error (MSE) by the end of

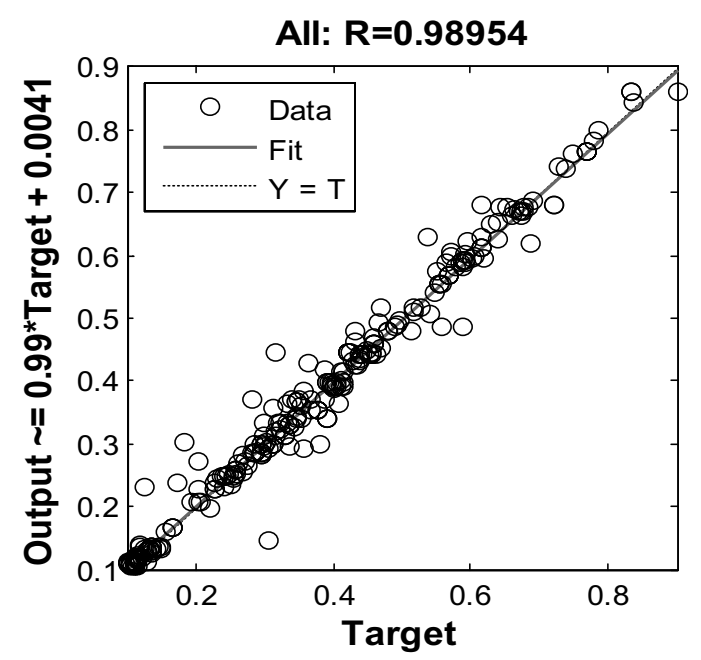

Fig. 4. Regression plot for ANN architecture 4-7-5-13-1. the training process is 0.00069 . The best validation performance is achieved at 75th epoch.

Regression plot for this architecture is shown in Fig. 4. The value of $R$ in this case is found to be 0.98954 .

\section{Performance Comparison}

For the purpose of comparison, measured values of breakdown strength and predicted values of breakdown strength (ANN) are shown on the same graph (Fig. 5).

An equation was developed in Ref. 6 using an empirical relation suggested by Swanson and Dall in Ref. 4 for the same cellulosic materials to predict the breakdown strength. The result reported in that study is also mentioned in the graph to compare both the prediction techniques. It can be seen from the graph (Fig. 5) that breakdown strength predicted by ANN is closer to the measured values for most of the test specimen considered, almost overlapping the measured value curve.

In Table 2, percentage errors between measured and predicted values of breakdown strength are tabulated. It is observed from the table that maximum error is $4.22 \%$ and the MAE is 1.2. Maximum percentage error observed while predicting the breakdown strength using empirical relation was $6.98 \%$. The difference in error values is shown in Fig. 6.

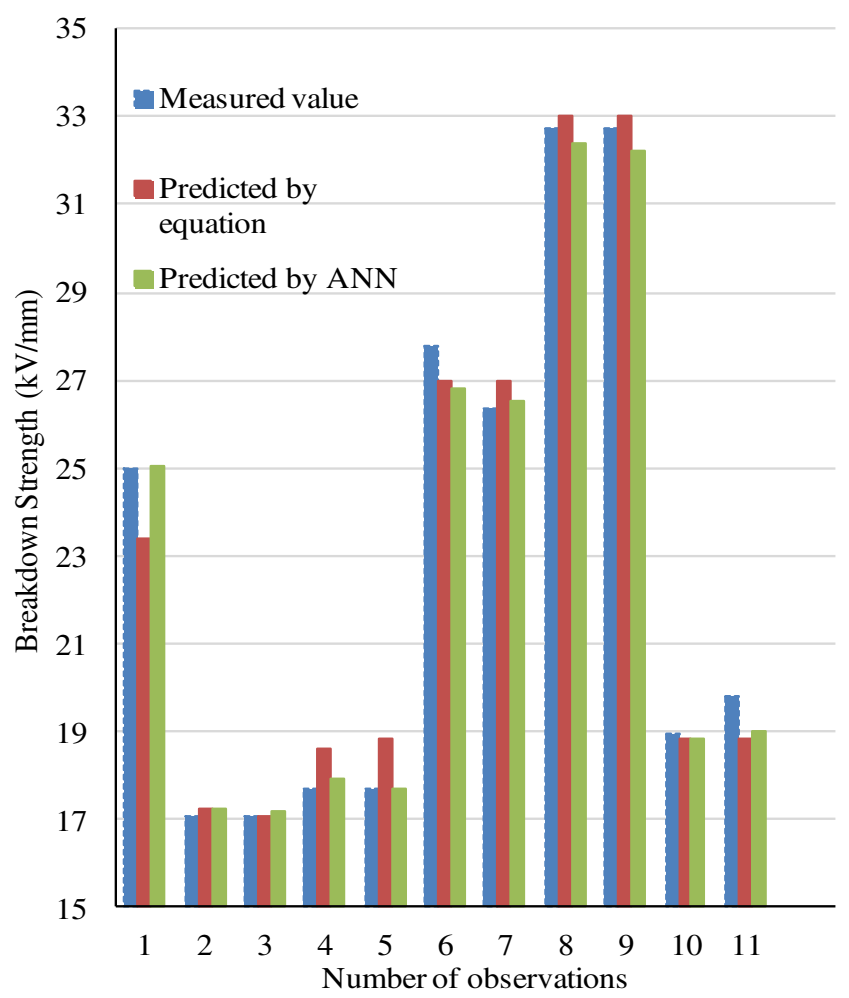

Fig. 5. Comparison of breakdown strength predicted by ANN and empirical relation with measured breakdown strength for different cellulosic materials. 
Table 2. Measured and predicted breakdown strength and error between them.

\begin{tabular}{|c|c|c|c|c|}
\hline S. No. & Cellulosic material & $\begin{array}{c}\text { Measured } \\
\text { BDS } \\
(\mathrm{kV} / \mathrm{mm})\end{array}$ & $\begin{array}{c}\text { Calculated } \\
\text { BDS } \\
(\mathrm{kV} / \mathrm{mm})\end{array}$ & $\%$ Error \\
\hline (1) & Manila paper & 25.00 & 25.03 & -0.11 \\
\hline (2) & Leatheroid paper & $\begin{array}{l}17.07 \\
17.07\end{array}$ & $\begin{array}{l}17.24 \\
17.17\end{array}$ & $\begin{array}{l}-0.96 \\
-0.59\end{array}$ \\
\hline (3) & Diamond-dotted paper & $\begin{array}{l}17.70 \\
17.70\end{array}$ & $\begin{array}{l}17.95 \\
17.67\end{array}$ & $\begin{array}{c}-1.38 \\
0.17\end{array}$ \\
\hline (4) & Paper phenolic sheet & $\begin{array}{l}27.75 \\
26.36\end{array}$ & $\begin{array}{l}26.79 \\
26.51\end{array}$ & $\begin{array}{c}3.58 \\
-0.57\end{array}$ \\
\hline (5) & Cotton phenolic sheet & $\begin{array}{l}32.71 \\
32.71\end{array}$ & $\begin{array}{l}32.39 \\
32.19\end{array}$ & $\begin{array}{l}0.98 \\
1.60\end{array}$ \\
\hline \multirow[t]{2}{*}{ (6) } & Presspaper & $\begin{array}{l}18.93 \\
19.80\end{array}$ & $\begin{array}{l}18.86 \\
19.00\end{array}$ & $\begin{array}{l}0.38 \\
4.22\end{array}$ \\
\hline & & & MAE & 1.29 \\
\hline
\end{tabular}

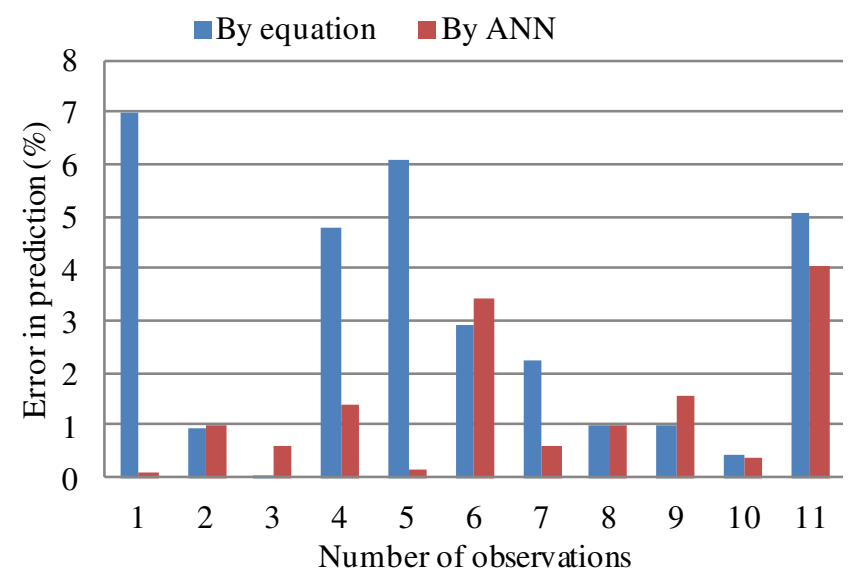

Fig. 6. Comparison of error in prediction of breakdown strength by ANN and empirical equation.

\section{Conclusions}

In this work, different electrical parameters of cellulosic insulating materials are studied. Breakdown strength of these materials is measured experimentally as well as using a nature-inspired computational technique known as ANN considering that it is dependent on other parameters like thickness of insulation sample, volume resistivity, relative permittivity, and loss tangent. Different ANN models are trained and a best fit model having minimum MAE between the measured and the predicted values of breakdown strength is selected. A model with 4 number of neurons in input layer, 7,5 , and 13 neurons in 3 hidden layers, and 1 output layer neuron (4-7-5-13-1) has shown the best performance with the lowest MAE, that is, 1.29. Breakdown strength predicted for the same insulating materials using an empirical relation suggested by Swanson and Dall was also considered to compare the results with the ANN model proposed in this work. Results observed from ANN modeling are found in good agreement with the measured values. Also, the errors are much lower when compared to the errors obtained with empirical relation.

It is expected that the proposed model will be helpful for practicing engineers for assessment of breakdown strength for cellulosic insulating materials.

\section{References}

${ }^{1}$ K. P. Wong, Electrical Engineering, Vol. 2 (ELOSS Publishers/ UNESCO, UK, 2009), pp. 42-53.

${ }^{2}$ M. Schwartz, Encyclopaedia of Materials, Parts and Finishes, 2nd edn. (CRC press, London, 2002), pp. 190-192.

${ }^{3}$ F. Cardarelli, Materials Handbook: A Concise Desktop Reference, 2nd edn. (Springer Science \& Business Media, London, 2008), pp. 519-530.

${ }^{4}$ J. W. Swanson and F. C. Dall, On the dielectric strength of synthetic electrical insulating materials, IEEE Trans. Electr. Insul. 12, 2 (1977).

${ }^{5}$ A. Masood, M. U. Zuberi and E. Husain, Breakdown strength of solid dielectrics in liquid nitrogen, IEEE Trans. Dielectr. Electr. Insul. 15, 4 (2008).

${ }^{6}$ S. Singh, M. M. Mohsin and A. Masood, Diagnosis of present-day cellulosic insulating materials used in electrical power industry, IEEE 7th Power India Int. Conf. (Bikaner, India, 2016).

${ }^{7}$ E. Husain, M. M. Mohsin and R. S. Nema, On electric strength of solid insulating materials, IEEE Conf. Electrical Insulation and Dielectric Phenomena (Leesburg, Virginia, 1989), pp. 453-458.

${ }^{8}$ DuPont, Nomex paper type 410 , technical data sheet, $K$-20612-1 (USA, 2013).

${ }^{9}$ A. Von Hippel, Dielectric Materials and Applications (The Technology Press of MIT, Wiley, 1954).

${ }^{10}$ E. W. Washburn, International Critical Tables of Numerical Data, Physics, Chemistry and Technology, Vol. 2 (National Academy of Science, USA, 1927).

${ }^{11}$ ASTM D150-98, Standard test methods for ac loss characteristics and permittivity (dielectric constant) of solid electrical insulation (ASTM international designation, West Conshohocken, PA, 2004), p. 3.

${ }^{12}$ Automatic Capacitance \& Dissipation Factor (Tan-Delta) Test System (Model PE-ACDF-1), Operation Manual, Power Electronical (Nasik, India).

${ }^{13}$ S. Singh, M. M. Mohsin and A. Masood, High voltage dielectric diagnosis using automatic capacitance \& dissipation factor test system, IEEE 1st Int. Conf. Power Electronics, Intelligent Control and Energy Systems (New Delhi, India, 2016), pp. 1-4.

${ }^{14}$ ASTM D149-09, Standard Test Method for Dielectric Breakdown Voltage and Dielectric Strength of Solid Electrical Insulating Materials at Commercial Power Frequencies (ASTM International, West Conshohocken, PA, 2013), p. 2.

${ }^{15}$ IEEE guide for the statistical Analysis of electrical insulation breakdown data, IEEE standard 930-2004 (revision of IEEE standard 930-1987, IEEE, 2005), p. 1.

${ }^{16}$ S. Hykin, Neural Networks: A Comprehensive Foundation, 2nd edn. (Pearson Prentice Hall, New Jersey, USA, 2005).

${ }^{17}$ H. Demuth and M. Beale, Neural Network Toolbox User's Guide (The MathWorks Inc. online edition, Natick, MA, 2002). 